\title{
System size dependence of strange particle yields and spectra at $\sqrt{s_{N N}}=17.3 \mathrm{GeV}$
}

\author{
Ingrid Kraus for the NA49 Collaboration \\ Gesellschaft für Schwerionenforschung (GSI), Darmstadt, Germany
}

\begin{abstract}
Yields and spectra of strange hadrons $\left(\mathrm{K}^{+}, \mathrm{K}^{-}, \phi, \Lambda\right.$ and $\left.\bar{\Lambda}\right)$ as well as of charged pions were measured in near central $\mathrm{C}+\mathrm{C}$ and $\mathrm{Si}+\mathrm{Si}$ collisions at $158 \mathrm{~A}$ $\mathrm{GeV}$ beam energy with the NA49 detector. Together with earlier data for $\mathrm{p}+\mathrm{p}, \mathrm{S}+\mathrm{S}$ and $\mathrm{Pb}+\mathrm{Pb}$ reactions the system size dependence can be studied. Relative strangeness production rises fast and saturates at about 60 participating nucleons; the net hyperon spectra show an increasing shift towards midrapidity for larger colliding nuclei. An interpretation based on the formation of coherent systems of increasing volume is proposed. The transverse mass spectra can be described by a blast wave ansatz. Increasing flow velocity is accompanied by decreasing temperatures for both kinetic and chemical freeze out. The increasing gap between inelastic and elastic decoupling leaves space for rescattering.
\end{abstract}

PACS numbers: 25.75.-q

\section{Introduction}

The production of strange particles in heavy ion collisions is studied in many experiments since it had been proposed as a signature for the transition to a deconfined state of strongly interacting matter [1]. Although enhanced strangeness production in $A+A$ collisions relative to elementary reactions is observed over a wide range of c.m. energy [2], the question about its origin is still unsolved. This motivated the systematic study of symmetric collisions of nuclei with increasing mass number $A$.

The presented data were recorded with the NA49 hadron spectrometer [3] at the CERN SPS. The analysis of meson $\left(\pi^{ \pm}, \mathrm{K}^{ \pm}, \phi\right)$ and $\Lambda$ hyperon production is described in [4], the preliminary results for $\bar{\Lambda}$ baryons were obtained analogously.

\section{Results}

The yields of all particles under study were measured in $\left(y, p_{T}\right)$ bins, the transverse mass $\left(m_{T}=\sqrt{m_{0}^{2}+p_{T}^{2}}\right)$ spectra at midrapidity are shown in figure 1 (left). Fits with the thermal ansatz

$$
\frac{1}{m_{T}} \cdot \frac{\mathrm{d}^{2} n}{\mathrm{~d} y \cdot \mathrm{d} m_{T}}=\mathrm{c} \cdot \mathrm{e}^{-m_{T} / T}
$$



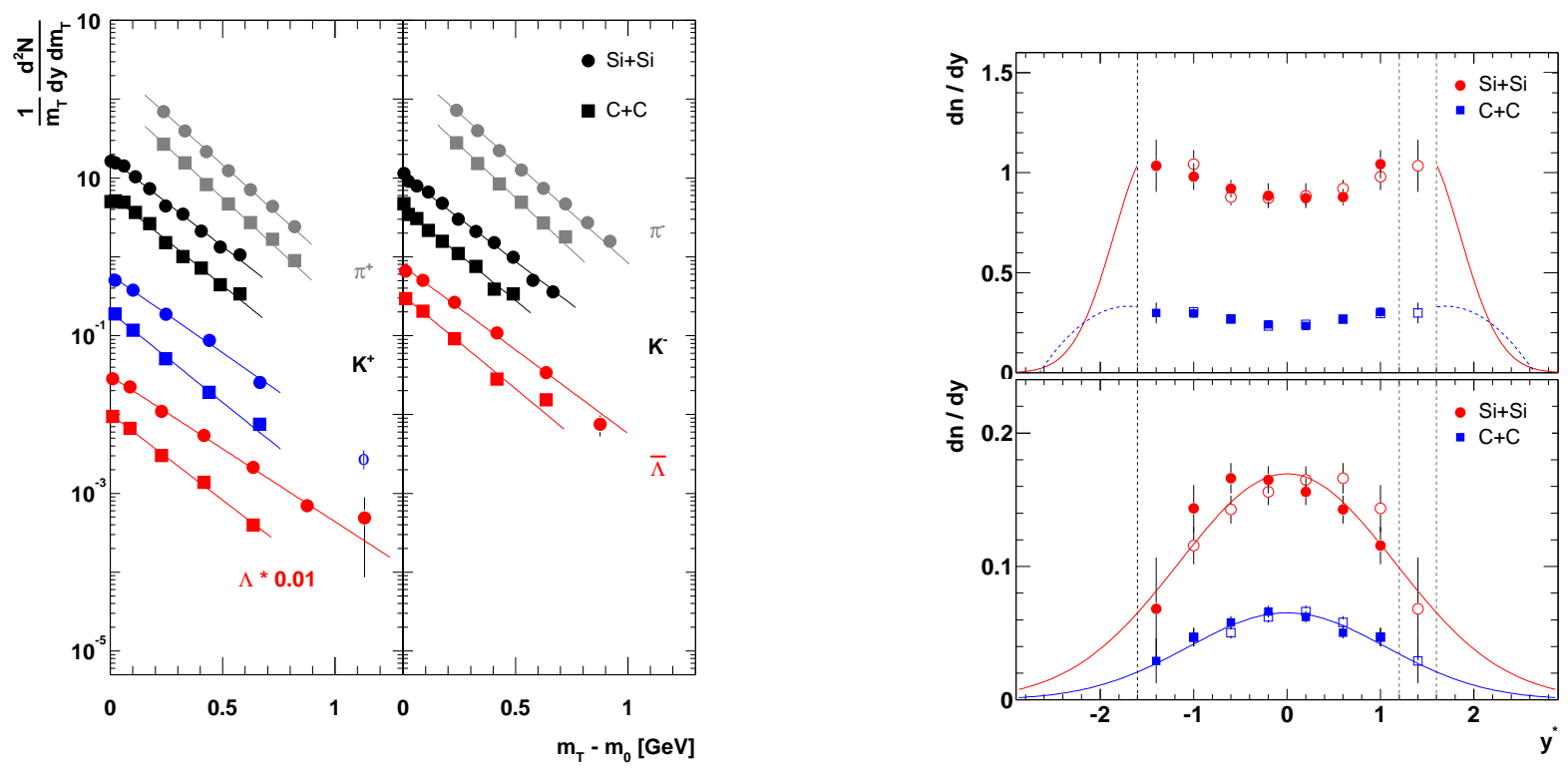

Figure 1. Left: transverse mass spectra of various particles, measured at midrapidity in $\mathrm{C}+\mathrm{C}(\boldsymbol{\square})$ and $\mathrm{Si}+\mathrm{Si}(\bullet)$ reactions. Shown are the exponential fits with equation 1. Right: rapidity spectra of $\Lambda$ (top) and $\bar{\Lambda}$ hyperons (bottom, preliminary). Only statistical errors are included.

were used to extrapolate to $p_{T}$ regions not covered by the measurement. The rapidity densities $\mathrm{d} n / \mathrm{d} y$ of the hyperons are shown in figure 1 (right), while the mesons are presented in [4]. For the extrapolation to the very forward region the pion rapidity distributions were approximated by a superposition of two Gaussians displaced symmetrically around midrapidity, whereas single Gaussians were used for the other mesons and $\bar{\Lambda}$ hyperons. To obtain the yields of $\Lambda$ hyperons their rapidity distributions were extrapolated by shapes adopted from $\mathrm{p}+\mathrm{p}$ and $\mathrm{S}+\mathrm{S}$ measurements. The mean of both approximations was taken for $\mathrm{C}+\mathrm{C}$, the shape from $\mathrm{S}+\mathrm{S}$ was used for $\mathrm{Si}+\mathrm{Si}$ (lines in figure 1).

\section{Discussion}

\subsection{Strange particle production}

The strange particle yield per pion is shown in figure 2 together with data from $\mathrm{p}+\mathrm{p}$ and $\mathrm{Pb}+\mathrm{Pb}$ collisions [5] measured by the NA49 Collaboration and $\mathrm{S}+\mathrm{S}$ results [6] from NA35. All presented data on pions and hyperons are corrected for feed down contaminations. The mean number of nucleons in the geometrical overlap area $\left\langle N_{W}\right\rangle$ serves as a measure of the system size. The ratio of strange hadrons to pions exhibits for all considered particles a fast rise in small systems and reaches the level of $\mathrm{Pb}+\mathrm{Pb}$ interactions at about 60 wounded nucleons.

Empirical scaling parameters as e.g. the nucleon or collision density [7] suggest that the density reached in $\mathrm{A}+\mathrm{A}$ interactions has an impact on the strangeness production. 


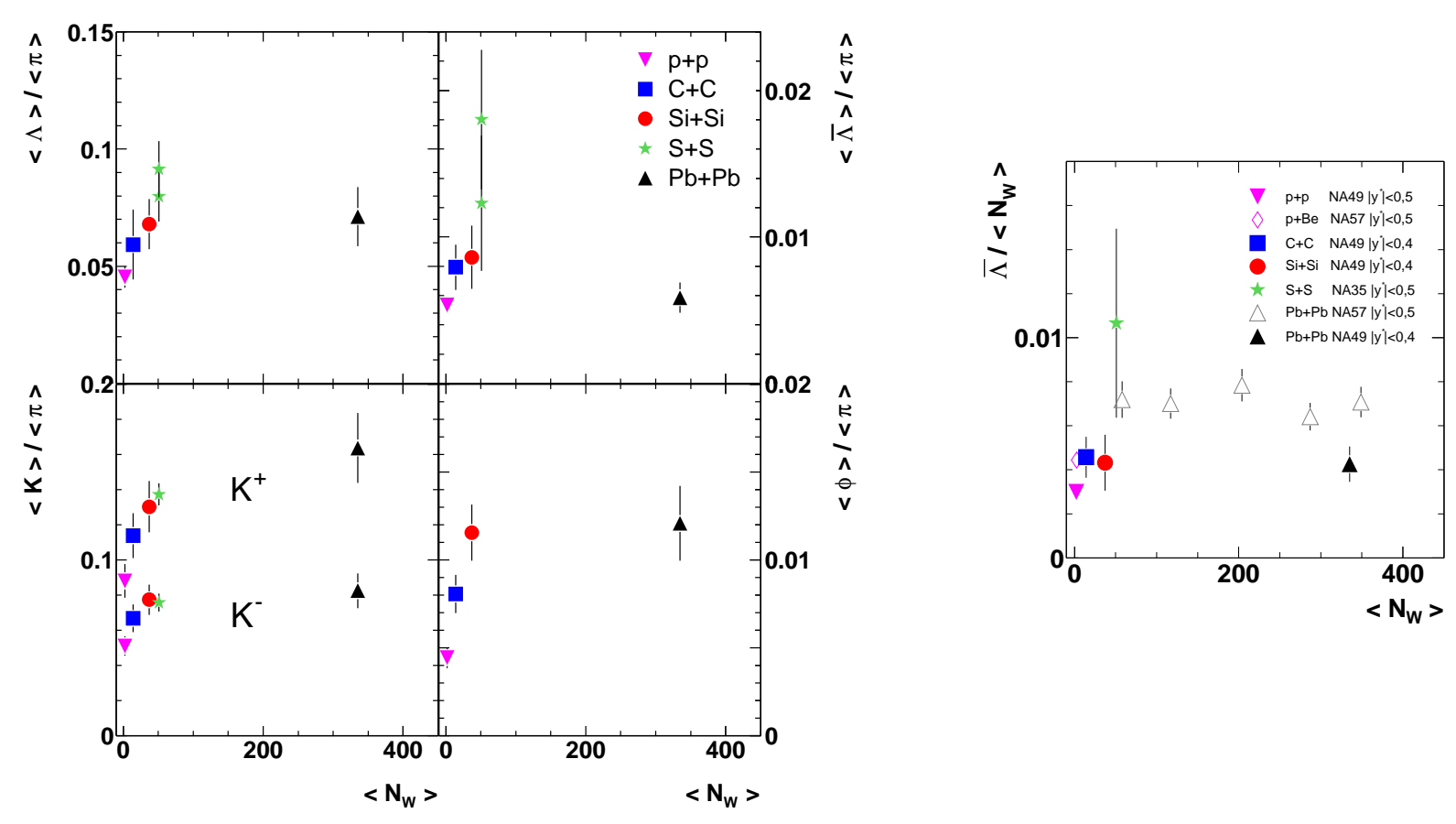

Figure 2. Left: multiplicity of hyperons, charged kaons and $\phi$ mesons per pion, $\langle\pi\rangle=\frac{1}{2}\left(\left\langle\pi^{+}\right\rangle+\left\langle\pi^{-}\right\rangle\right)$. Right: $\bar{\Lambda}$ density at midrapidity per wounded nucleon in $\mathrm{p}+\mathrm{p}$ $(\mathbf{v}), \mathrm{p}+\mathrm{Be}(\diamond), \mathrm{C}+\mathrm{C}(\boldsymbol{\square}), \mathrm{Si}+\mathrm{Si}(\bullet)$, minimum bias $(\triangle)$ and central $\mathrm{Pb}+\mathrm{Pb}(\boldsymbol{\Delta})$ collisions at $158 A \mathrm{GeV}$ as well as in $\mathrm{S}+\mathrm{S}$ reactions $(\star)$ at $200 A \mathrm{GeV}$. The error bars represent the squared sum of statistical and systematic errors.

At sufficiently high density one might expect that the subsequent collisions do not occur independently anymore, but that connected domains of overlapping resonances or strings are created. These volumes can become quantum-mechanically coherent and decay as objects which can be considerably larger than those created in isolated $\mathrm{p}+\mathrm{p}$ interactions.

The volume dependence of strangeness production is described in statistical models by the transition from canonical to grand canonical ensembles. The calculation by Tounsi and Redlich [8] agrees qualitatively with the measurements presented in figure 2, but quantitatively the saturation level is reached for a distinctly smaller number of participants in the model. Theory and experiment might be reconciled by two plausible modifications: assuming that only parts of the reaction volume that is spread over about 3 units of rapidity [9] are coherent or that the linear relationship between volume and number of participants used to link model and measurement has to be refined.

\subsection{Chemical freeze out conditions}

The measured particle yields can be reproduced by the statistical hadronisation model of Becattini [10], see figure 3. The strangeness production in the corresponding equilibrated resonance gas is suppressed $\left(\gamma_{S}<1\right)$. This suppression diminishes with increasing system size (figure 4), following the observed strangeness enhancement. The 

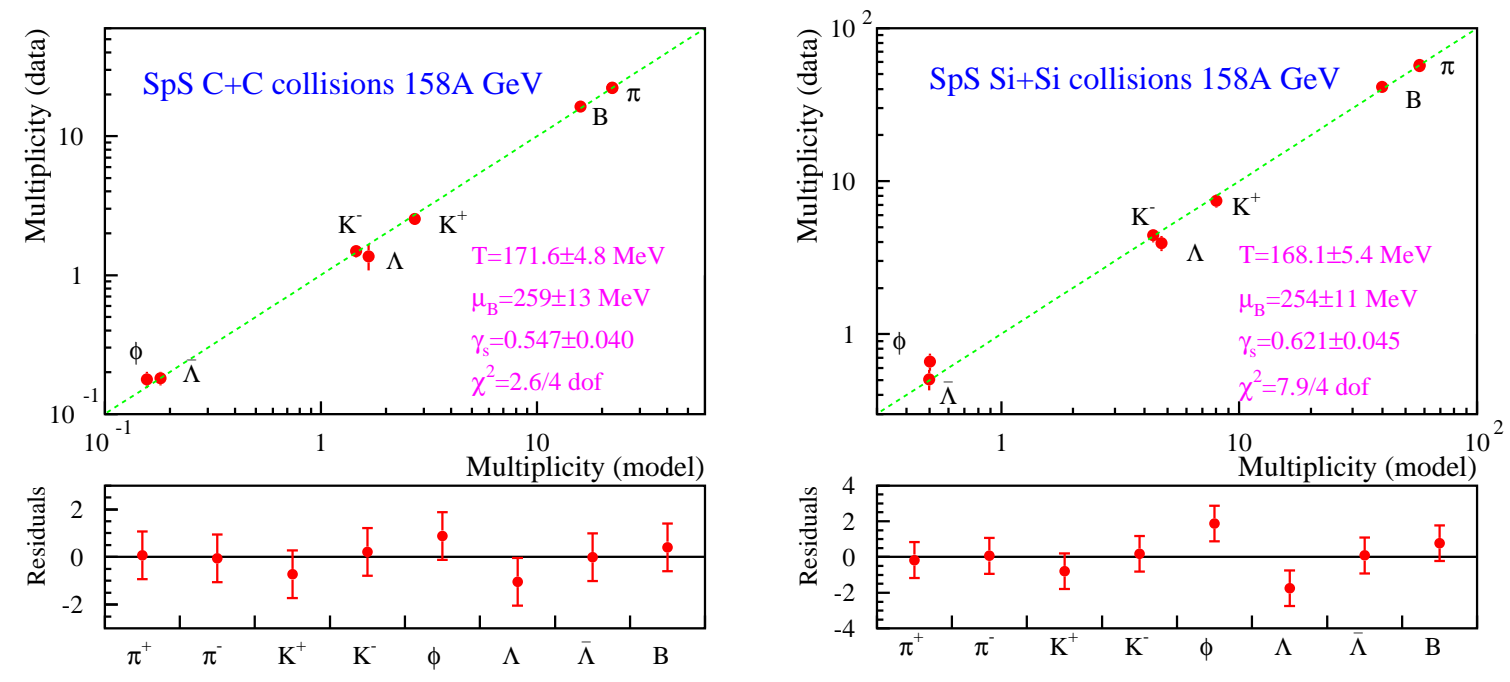

Figure 3. Comparison of the measured preliminary particle yields in $\mathrm{C}+\mathrm{C}$ (left) and $\mathrm{Si}+\mathrm{Si}$ collisions (right) to calculations with the statistical hadronisation model [10].

baryochemical potential $\mu_{B}$ does not depend significantly on the system size, while the decoupling temperature $T_{c h}$ is higher in smaller reaction volumes. This would leave less room for inelastic rescattering in the small systems, if the varying $T_{c h}$ is not entirely provoked by the changing $\gamma_{S}$ as suggested in [14]. The freeze out points of $\mathrm{C}+\mathrm{C}$ and $\mathrm{Si}+\mathrm{Si}$ reactions appear in the QCD phase diagram in the vicinity of the phase boundary as seen in figure 4.

\subsection{Absorption of $\bar{\Lambda}$ hyperons}

The increasing absorption of anti-baryons $(\bar{p}$ and $\bar{d})$ with increasing centrality of $\mathrm{Pb}+\mathrm{Pb}$ reactions at $158 \mathrm{AGeV}$ was observed in a forward rapidity window ( $\mathrm{y}=3.7$ ) by the NA52 Collaboration [23] and explained with an increasing baryochemical potential. In contrast to that, the $\langle\bar{\Lambda}\rangle$ yield per pion in central $\mathrm{Pb}+\mathrm{Pb}$ collisions is not significantly smaller than in $\mathrm{Si}+\mathrm{Si}$ reactions (figure 2 left), while the midrapidity densities are on the same level. Moreover the measurement by the NA57 Collaboration [24] indicates even a further increase from central $\mathrm{Si}+\mathrm{Si}$ to $\mathrm{Pb}+\mathrm{Pb}$ data at different centralities (figure 2 right). This is in accord with the almost constant $\mu_{B}$ shown in figure 4.

\subsection{Particle ratios}

Both, the $\left\langle\mathrm{K}^{-}\right\rangle /\left\langle\mathrm{K}^{+}\right\rangle$as well as the $\langle\bar{\Lambda}\rangle /\langle\Lambda\rangle$ ratio (figure 5 ) of the total yields exhibit no significant dependence on the system size. Due to that, the strange hadron potential $\mu_{S}$ calculated (with the strange quark potential $\mu_{s}$ ) as [25]

$$
\mu_{S}=\frac{1}{3} \mu_{B}-\mu_{s} \quad \text { with } \quad \frac{\langle\Lambda\rangle}{\langle\bar{\Lambda}\rangle} \cdot\left(\frac{\left\langle K^{-}\right\rangle}{\left\langle K^{+}\right\rangle}\right)^{2}=\exp \left(6 \cdot \frac{\mu_{s}}{T}\right)
$$



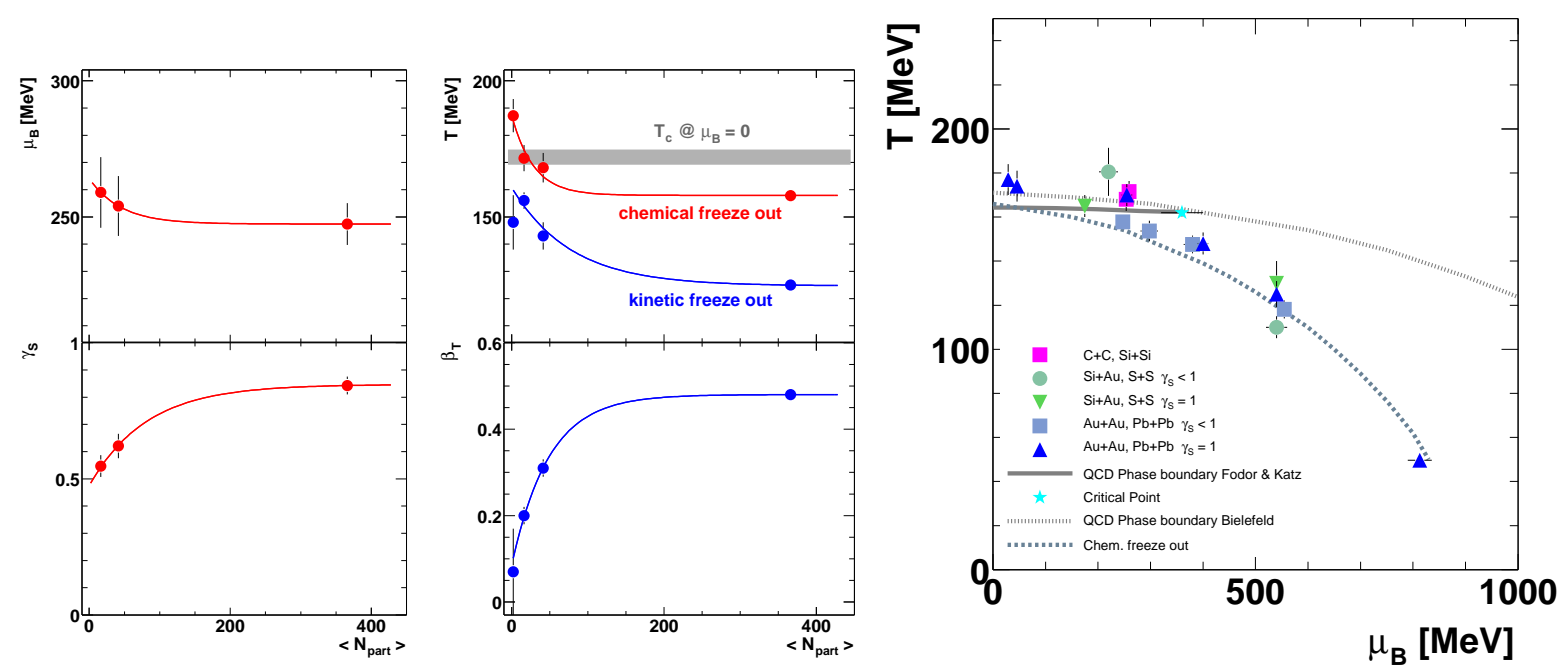

Figure 4. Left: chemical freeze out conditions for $\mathrm{C}+\mathrm{C}, \mathrm{Si}+\mathrm{Si}, \mathrm{Pb}+\mathrm{Pb}$ at $158 \mathrm{~A}$ $\mathrm{GeV}$ from fits with the statistical hadronisation model $[10,11]$. The curves are shown to guide the eye and represent a functional form $f\left(\left\langle N_{\text {part }}\right\rangle\right)=a+b \cdot \exp \left(c \cdot\left\langle N_{\text {part }}\right\rangle\right)$. Middle: freeze out conditions for $\mathrm{p}+\mathrm{p}, \mathrm{C}+\mathrm{C}, \mathrm{Si}+\mathrm{Si}, \mathrm{Pb}+\mathrm{Pb}$ at $158 A \mathrm{GeV}$. The temperatures at chemical decoupling are from fits with the statistical hadronisation model $[10,11]$. The kinetic freeze out temperature and the transverse flow velocity $\beta_{T}$ are from blast wave fits to the transverse mass spectra, equation 3 . The values for $\mathrm{p}+\mathrm{p}$ and $\mathrm{Pb}+\mathrm{Pb}$ interaction were taken from [12]. The critical temperature $\mathrm{T}_{c}$ is from lattice QCD calculations [13].

Right: QCD phase diagram. The phase boundary between deconfined and hadronic matter was estimated with lattice calculations by the Bielefeld [15] as well as by the Budapest group [16]. The parametrisation of the chemical freeze out points as a curve of constant energy per hadron of $\langle E\rangle /\langle N\rangle=1 \mathrm{GeV}$ is taken from [17]. The $\mathrm{C}+\mathrm{C}$ and $\mathrm{Si}+\mathrm{Si}$ data [10] lie between the $\mathrm{Si}+\mathrm{Au}$ measurements at the AGS at $14.6 \mathrm{AGeV}$ and the $\mathrm{S}+\mathrm{S}$ data at $200 \mathrm{~A} \mathrm{GeV}$. Model calculations with $\gamma_{S}=1$ are from [18], those with free $\gamma_{S}$ are from [19]. The freeze out points determined from the fits to the $\mathrm{Au}+\mathrm{Au}$ and $\mathrm{Pb}+\mathrm{Pb}$ data with $\gamma_{S}=1$ from SIS [20], AGS [18] and SPS [21] up to RHIC energies [22] agree with those from the fits with free $\gamma_{S}$ for the AGS and SPS [11].
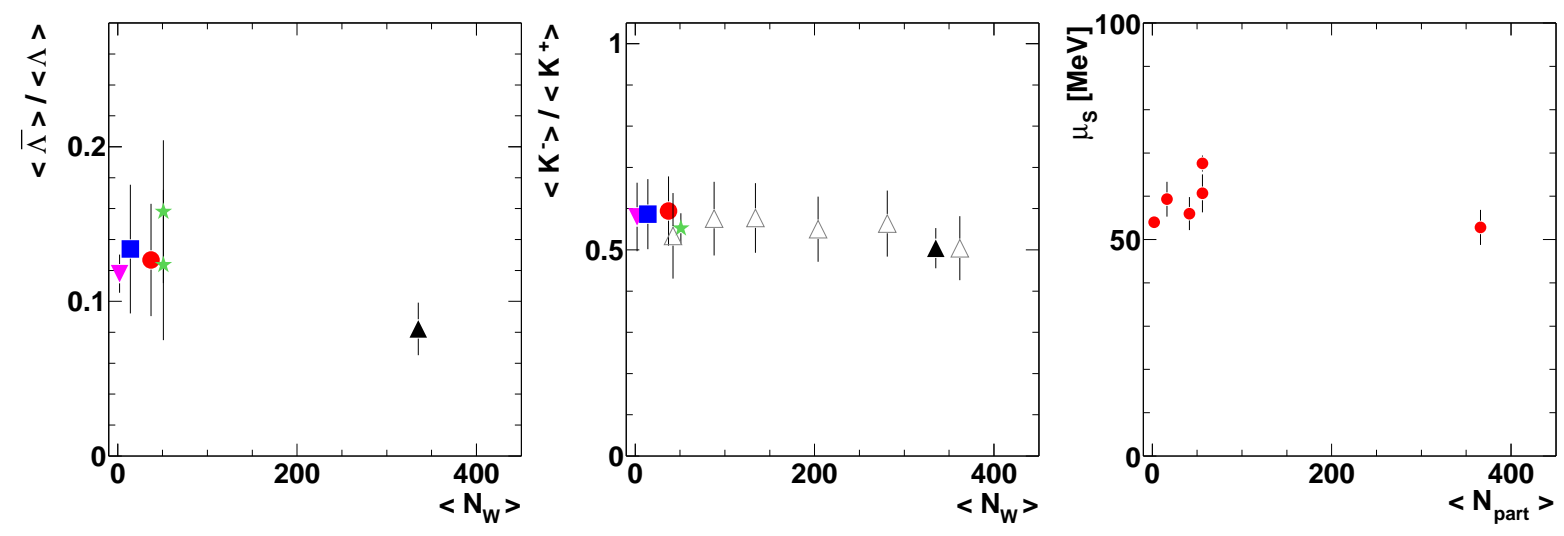

Figure 5. $\langle\bar{\Lambda}\rangle /\langle\Lambda\rangle$ (left) and $\left\langle\mathrm{K}^{-}\right\rangle /\left\langle\mathrm{K}^{+}\right\rangle$ratio (middle) of the total yields measured in $\mathrm{p}+\mathrm{p}(\boldsymbol{\nabla}), \mathrm{C}+\mathrm{C}(\mathbf{\square}), \mathrm{Si}+\mathrm{Si}(\bullet)$, minimum bias $(\triangle)$ and central $\mathrm{Pb}+\mathrm{Pb}(\boldsymbol{\Lambda})$ collisions at $158 A \mathrm{GeV}$ as well as in $\mathrm{S}+\mathrm{S}$ reactions $(\star)$ at $200 A \mathrm{GeV}$. The error bars represent the squared sum of statistical and systematic errors. The strange hadron potential $\mu_{S}$ (right) is calculated from these ratios. 

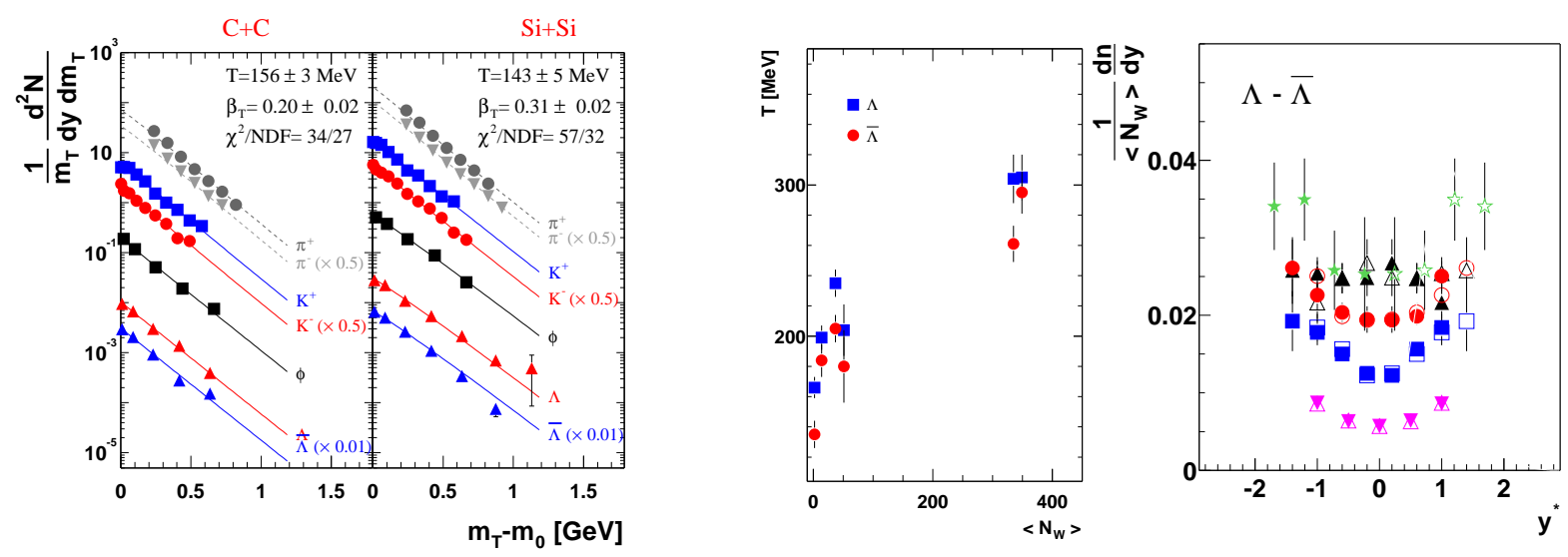

Figure 6. Left: transverse mass spectra of various particles from $\mathrm{C}+\mathrm{C}$ (left part) and $\mathrm{Si}+\mathrm{Si}$ collisions (right part) measured at midrapidity. Also shown are the fits with the blast wave model (equation 3).

Middle: Inverse slope parameters of $\Lambda(\boldsymbol{\square})$ and $\bar{\Lambda}(\bullet)$ hyperons (equation 1 ) in $\mathrm{p}+\mathrm{p}$, $\mathrm{C}+\mathrm{C}, \mathrm{Si}+\mathrm{Si}, \mathrm{S}+\mathrm{S}$ and $\mathrm{Pb}+\mathrm{Pb}$ collisions.

Right: net hyperon rapidity density normalised to the number of wounded nucleons $\left\langle\mathrm{N}_{W}\right\rangle$ measured in $\mathrm{p}+\mathrm{p}(\boldsymbol{\nabla}), \mathrm{C}+\mathrm{C}(\mathbf{\square}), \mathrm{Si}+\mathrm{Si}(\bullet)$ and $\mathrm{Pb}+\mathrm{Pb}(\boldsymbol{\Lambda})$ collisions at $158 \mathrm{~A}$ $\mathrm{GeV}$ as well as in $\mathrm{S}+\mathrm{S}$ reactions $(\star)$ at $200 A \mathrm{GeV}$. Only statistical errors are included.

is constant as well. In spite of zero net strangeness the potential is not vanishing, it amounts to about $60 \mathrm{MeV}$ (figure 5). This is in agreement with the statistical model prediction [26] for the fitted values of $T_{c h}$ and $\mu_{B}$.

\subsection{Net hyperon density}

The $\bar{\Lambda} / \Lambda$ ratio at midrapidity (not shown) is (in contrast to the flat $\langle\bar{\Lambda}\rangle /\langle\Lambda\rangle$ ratio) steeply decreasing with increasing system size for small reaction volumes, followed by a saturation above about 60 wounded nucleons. The ratio reflects directly the changing $\Lambda$ rapidity distribution while the shape of the $\bar{\Lambda}$ hyperon spectra remains the same. The difference of the two distributions, the net hyperon density, is shown in figure 6 . The flattening of the $(\Lambda-\bar{\Lambda})$ rapidity spectra with increasing system size can be understood in terms of stronger stopping due to an increasing number of collisions. This leads to a successive shift of the incoming baryons from beam towards mid rapidity. Thereby the energy per nucleon deposited in the fireball is increasing and more energy for the excitation of resonances or strings is provided.

\subsection{Transverse mass spectra}

The inverse slope parameters $T$ from exponential fits to the $m_{T}$ spectra with equation 1 are systematically higher for $\Lambda$ than for $\bar{\Lambda}$ hyperons as can be seen from figure 6 . In small systems this may be traced back to the anti-correlation of the (higher $\bar{\Lambda}$ ) production threshold on one hand and the kinetic energy of the created particles on the other hand due to energy conservation [27]. The cause in the large systems is not clear. 
The slopes of both, $\Lambda$ and $\bar{\Lambda}$ hyperons, fit into the picture of growing radial flow with increasing system size. Moreover the $m_{T}$ spectra can be approximated with a hydrodynamical model; here a simplified version from Schnedermann et al. [28] with constant flow velocity $\beta_{T}$ is utilised to fit all particles except the pions simultaneously with the function

$$
\frac{\mathrm{d} n}{m_{T} \cdot \mathrm{d} m_{T}} \propto m_{T} \cdot \mathrm{I}_{0}\left(\frac{p_{T} \cdot \sinh \rho}{T}\right) \cdot \mathrm{K}_{1}\left(\frac{m_{T} \cdot \cosh \rho}{T}\right),
$$

where $\mathrm{I}_{0}$ and $\mathrm{K}_{1}$ are modified Bessel functions and $\rho$ includes $\beta_{T}: \rho=\operatorname{atanh} \beta_{T}$. The fit to the $\mathrm{C}+\mathrm{C}$ and $\mathrm{Si}+\mathrm{Si}$ data is shown in figure 6 , while the system size dependence of the kinetic freeze out temperature and flow velocity $\beta_{T}$ is displayed in figure 4 . The $\mathrm{p}+\mathrm{p}$ and $\mathrm{Pb}+\mathrm{Pb}$ spectra were fitted with the same formula. In $\mathrm{p}+\mathrm{p}$ reactions $\beta_{T}$ is compatible with zero; in larger systems $\beta_{T}$ steeply increases. The increasing radial flow is accompanied by a decreasing kinetic freeze out temperature; $T_{k i n}$ drops by $30 \mathrm{MeV}$ between $\mathrm{C}+\mathrm{C}$ and $\mathrm{Pb}+\mathrm{Pb}$ collisions. Thereby the gap between chemical and thermal decoupling temperatures $\Delta T$ increases with size of the colliding nuclei, so more rescattering is expected in the larger systems.

The largest $\Delta T$ is observed in $\mathrm{p}+\mathrm{p}$ interactions, where neither rescattering nor flow is expected. This questions the mechanism described above as the only source of the effect. The broadening of $p_{T}$ spectra is already seen in $\mathrm{p}+\mathrm{A}$ data and multiple scattering is given there as an alternative explanation [29]. Furthermore there are models which describe the measured yields and spectra reasonably well by a single freeze out, e.g. [30].

\section{Summary}

Yields and spectra of strange hadrons and charged pions in $\mathrm{p}+\mathrm{p}, \mathrm{C}+\mathrm{C}, \mathrm{Si}+\mathrm{Si}, \mathrm{S}+\mathrm{S}$ and $\mathrm{Pb}+\mathrm{Pb}$ collisions were presented. The fast rise of the relative strangeness production, followed by a saturation above about 60 participating nucleons, together with the increasing shift of net hyperons towards mid rapidity with increasing size of the colliding nuclei suggests the creation of coherent domains as a possible interpretation. The hadronisation of the small systems occurs in the vicinity of the phase boundary. No significant absorption of $\bar{\Lambda}$ hyperons is seen. The transverse mass spectra can be described by a blast wave ansatz. Fits to the data indicate increasing flow velocity accompanied by decreasing temperatures for both kinetic and chemical freeze out. The increasing gap between inelastic and elastic decoupling in $\mathrm{A}+\mathrm{A}$ collisions leaves space for rescattering.

[1] Rafelski J and Müller B 1982 Phys. Rev. Lett. 481066 Koch P, Müller B and Rafelski J 1986 Phys. Rep. 142167

[2] Ritter H G and Wang X (editors) 2004 Quark Matter 2004 (Institute of Physics Publishing)

[3] Afanasiev S V et al (NA49 Collaboration) 1999 Nucl. Instr. Meth. A 430210

[4] Alt C et al (NA49 Collaboration) Preprint nucl-ex/0406031 
[5] Sikler F et al (NA49 Collaboration) 1999 Nucl. Phys. A 661 45c

Afanasiev S V et al (NA49 Collaboration) 2000 Phys. Lett. B 49159

Afanasiev S V et al (NA49 Collaboration) 2002 Phys. Rev. C 66054902

Susa $\mathrm{T}$ et al (NA49 Collaboration) 2002 Nucl. Phys. A 698 491c

Barna D 2002 Ph.D. thesis, University of Budapest

Höhne C 2003 Ph.D. thesis, University of Marburg http://archiv.ub.uni-marburg.de/diss/z2003/0627/

Anticic T et al (NA49 Collaboration) 2004 Phys. Rev. Lett. 93022302

[6] Bartke J et al (NA35 Collaboration) 1990 Z. Phys. C 48191

Bächler J et al (NA35 Collaboration) 1993 Z. Phys. C 58367

Alber T et al (NA35 Collaboration) 1994 Z. Phys. C 64195

Alber T et al (NA35 Collaboration) Preprint hep-ex/9711001

[7] Bialkowska H and Retyk W 2001 J. Phys. G 27397

Höhne C et al (NA49 Collaboration) 2003 Nucl. Phys. A 715 474c

[8] Tounsi A and Redlich K 2002 J. Phys. G 282095

[9] Appelshäuser H. et al (NA49 Collaboration) 1999 Phys. Rev. Lett. 822471

[10] Becattini F 2004 private communication

[11] Becattini F et al 2004 Phys. Rev. C 69024905

[12] van Leeuwen M et al (NA49 Collaboration) 2003 Nucl. Phys. A 715 161c van Leeuwen M 2004 private communication

[13] Fodor Z and Katz S D 2002 JHEP 0203014 (Preprint hep-lat/ 0106002)

[14] Cleymans et al Preprint hep-ph/0409071

[15] Allton C R et al 2002 Phys. Rev. D 66074507

[16] Fodor Z and Katz S D Preprint hep-lat/0402006

[17] Cleymans J and Redlich K 1998 Phys. Rev. Lett. 815284

[18] Braun-Munzinger P et al 1996 Phys. Lett. B 3651

Braun-Munzinger P et al 1995 Phys. Lett. B 34443

[19] Becattini F et al 1998 Eur. Phys. J. C 5143

Cleymans J et al 1997 Z. Phys. C 74319

[20] Becattini F et al 2001 Phys. Rev. C 64024901

[21] Braun-Munzinger P, Heppe I and Stachel J 1999 Phys. Lett. B 46519

[22] Braun-Munzinger P et al 2001 Phys. Lett. B 51841

[23] Ambrosini G et al (NA52 Collaboration) 1999 New J. Phys. 122.1

[24] Bruno G E et al (NA57 Collaboration) 2004 J. Phys. G $30 \mathrm{~S} 717$

[25] Sollfrank J et al 1994 Z. Phys. C 61659

[26] Braun-Munzinger P, Redlich K and Stachel J, Preprint nucl-th/ 0304013 to appear in Quark Gluon Plasma 3, eds. R.C. Hwa and X.N. Wang, World Scientific Publishing

[27] Kafka T et al 1977 Phys. Rev. D 161261

[28] Schnedermann E and Heinz U 1994 Phys. Rev. C 501675

[29] Fischer H G et al (NA49 Collaboration) 2003 Nucl. Phys. A $715118 \mathrm{c}$

[30] Letessier J et al 2001 J. Phys. G 27427

Broniowski W and Florkowski W 2002 Phys. Rev. Lett. 87272302 